\section{Psicologia Escolar \\ e Educacional}

ARTIGO

DOI: http://dx.doi.org/10.1590/2175-35392021224728

Localizador - e224728

\title{
CONSTRUÇÃO DE DEFINIÇÕES OPERACIONAIS EM METACOGNIÇÃO
}

\author{
Mauricio Abreu Pinto Peixoto ${ }^{1} \mathbb{D}$; Marcos Antônio Gomes Brandão ${ }^{1} \mathbb{D}$; Bruno Fragoso Tavares ${ }^{1} \mathbb{D}$
}

\section{RESUMO}

Desde a década de 70 a metacognição vem sendo entendida como "conhecimento e cognição sobre fenômenos cognitivos". Sua conceituação, apesar disso, ainda se apresenta inespecífica, a maioria dos pesquisadores tende a negligenciar o estabelecimento de definições mais precisas sobre a metacognição. Assim, foi realizada uma revisão narrativa, com enviesamento intencional orientado para a produção de definições operacionais aplicáveis ao processo de aprendizagem, de modo a permitir operar as ações de descrição, discriminação entre os diferentes fenômenos metacognitivos, sua classificação e interpretação de significados. A taxonomia aqui apresentada consta de 03 domínios (Habilidade, Experiência e Conhecimento Metacognitivos), trazendo 36 definições operacionais de termos metacognitivos.

Palavras-chave: metacognição; definição; processos cognitivos

\section{Construction of operational definitions in Metacognition}

\section{ABSTRACT}

Since the 70s, metacognition has been understood as "knowledge and cognition about cognitive phenomena". Its conceptualization, despite this, is still unspecific, most researchers tend to neglect the establishment of more precise definitions about metacognition. Thus, a narrative review was carried out, with an intentional bias oriented towards the production of operational definitions applicable to the learning process, in order to allow the operation of description actions, discrimination between different metacognitive phenomena, their classification and interpretation of meanings. The taxonomy presented here consists of 03 domains (Skill, Experience and Metacognitive Knowledge), bringing 36 operational definitions of metacognitive terms.

Keywords: metacognition; definition; cognitive processes

\section{Construcción de definiciones operacionales en Metacognición}

\section{RESUMEN}

Desde la década de 70 la metacognición sigue siendo entendida como "conocimiento y cognición sobre fenómenos cognitivos". Su conceptuación, a pesar de esto, aún se presenta inespecífica, la mayor parte de los investigadores tendiendo a la negligencia el establecimiento de definiciones más precisas sobre la metacognición. Así, se realizó una revisión narrativa, con desviación intencional orientado la a producción de definiciones operacionales aplicables al proceso de aprendizaje, de modo a permitir operar as acciones de descripción, discriminación entre los distintos fenómenos metacognitivos, su clasificación e interpretación de significados. La taxonomía aquí presentada consta de 03 dominios (Habilidad, Experiencia y Conocimiento Metacognitivos), trayendo 36 definiciones operacionales de términos metacognitivos.

Palabras clave: metacognición; definición; procesos cognitivos

\footnotetext{
${ }^{1}$ Universidade Federal do Rio de Janeiro - Rio de Janeiro - RJ - Brasil; geac.ufrj@gmail.com; marcosantoniogbrandao@gmail.com; brunnofragoso@gmail.com
} 


\section{INTRODUÇÃO}

Durante a década 70, Flavell propõe um novo termo - metacognição - para descrever o que ele definiu como "conhecimento e cognição sobre fenômenos cognitivos" (Flavell, 1976, p. 232). Desde então, as produções científicas têm se apresentado na descrição, explicação e experimentação desses componentes e, ainda mais, propondo outros elementos derivados da investigação da metacognição.

Pode-se afirmar que apesar da novidade do termo, o conceito em si é bem mais antigo. $O$ estudo seminal de Flavell foi precedido em conceitos tais como "monitoração da memória", "conhecimento sobre o conhecimento". Credita-se mesmo a Vygotsky (18961934) a paternidade do termo. Outros autores (Moritz \& Lysaker, 2018; Tarricone, 2011), buscando na reflexão base para a metacognição, leem textos de Aristóteles, Descartes, Spinoza e Santo Agostinho. Ainda mais, dada a natureza dinâmica das investigações em metacognição, torna-se apropriado considerar que é um conceito em desenvolvimento.

O interesse e a extensão da pesquisa metacognitivista podem ser demonstrados pelo elevado número de estudos realizados e pela diversidade de disciplinas envolvidas. Por exemplo, busca realizada no portal da CAPES incluindo apenas cinco anos (2014-2018) com o uso exclusivo do termo "metacognition" retornou 33.784 artigos. $O$ termo esteve associado a disciplinas das áreas das ciências da saúde, humanas, sociais aplicadas e linguísticas, letras e artes. Entretanto, parece existir dificuldades na constituição de definições que facilitem a agregação ou síntese de evidências de pesquisas.

Mesmo que os estudos lidem com o conceito, a maioria dos pesquisadores tende a negligenciar o estabelecimento de definições conceituais sobre a metacognição, muito provavelmente por ainda ser um conceito confuso (Gagnière, Betrancourt, \& Détienne, 2012; Scott \& Levy, 2013a). É duvidoso se o mais apropriado seria considerar a metacognição como um conceito guarda-chuva ou então como um conceito com fatores claros e distintos. Além disso, os autores apontam que utilizar múltiplos termos para expressar um mesmo conceito (ex.: habilidades executivas, crenças metacognitivas e julgamentos de aprendizagem) pode tornar a compreensão ainda mais vaga e confusa (Scott \& Levy, 2013a).

Entende-se que para descrever e interpretar fenômenos ou eventos metacognitivos na fala e demais formas de expressão de metacognição é necessário um rol de definições precisas e de preferência, operacionais. As definições conceituais (ou teóricas) fornecem o significado por definir um conceito em função de outros conceitos, consistindo das ideias, palavras e frases ou sentenças que apreendem possíveis significados e as definições operacionais estabelecem o significado em termos de observações e/ou atividades de medidas (Waltz, Strickland, \& Lenz, 2016). De certo modo, o avanço da pesquisa em metacognição depende do amplo acesso a definições operacionais que possam ser exploradas em estudos da área.

O foco do estudo orienta-se para a construção de definições operacionais em metacognição que permitam identificar a metacognição em discursos orais e escritos oriundos de pessoas envolvidas no processo de aprendizagem. Desse modo, o objetivo do artigo é apresentar definições de termos metacognitivos que possam ser aplicados a pesquisas do processo de aprendizagem humana. Nesse sentido, as definições aqui apresentadas constituem-se no resultado deste estudo.

\section{METODOLOGIA}

Revisão narrativa, com enviesamento intencional de artigos com definições de termos metacognitivos orientado para a produção de definições aplicáveis ao processo de aprendizagem. $O$ critério de escolha dos artigos foi sua capacidade em fornecer informação adequada para a construção de definições operacionais de termos metacognitivos. O componente conceitual da investigação foi desenvolvido a partir da construção de uma taxonomia de definições derivada de um mapeamento conceitual (não apresentado no artigo) que organizou elementos da metacognição produzindo uma estrutura de subsunção com as categorias dos constructos de habilidades metacognitivas, experiência metacognitiva e conhecimento metacognitivo e os 36 termos que the são subordinados. A escolha dessa técnica deveu-se ao seu caráter exploratório sob uma perspectiva fundamentalmente teórico-conceitual, sem a intenção de seleção sistemática das pesquisas (Cordeiro, Oliveira, Rentería, \& Guimarães, 2007; Rother, 2007).

Neste estudo o termo definição operacional foi entendido como aquele que "relaciona um conceito abstrato a eventos observáveis" (Cooper, 1982). Mais especificamente, esse foi utilizado para caracterizar uma definição que permite descrever e discriminar entre diferentes fenômenos metacognitivos, classificá-los e interpretar seus significados.

A coleta de artigos foi realizada em duas fases (Cooper, 1988; Pereira, 2012). Na primeira fase foi criada uma lista preliminar de autores e termos metacognitivos, além de estabelecer as bases técnicas para o estudo. O rol preliminar de termos e autores norteou a busca de artigos relevantes. Enfatize-se que tal lista não se constituiu como critério de exclusão de artigos; meramente visou alertar o analista para presença de artigos potencialmente relevantes. Essa fase foi realizada por um dos autores no Google Acadêmico e os artigos foram buscados por meio do rol de termos. A segunda fase, conduzida por outro pesquisador no Portal de Periódicos da CAPES, teve múltiplas etapas de filtragem reduzindo 
o volume inicial de 33.784 artigos para 189. Dentre os artigos, foram incluídos: (1) artigos originais, com foco teórico, de revisão ou empírico, nos quais se identificaram definições explícitas de termos metacognitivos e (2) publicados em periódicos avaliados por pares, entre os anos de 2014 e 2018.

Foram excluídos os artigos: (1) escritos em língua que não o inglês e o português e (2) os que apresentavam definições de terceiros quando o autor/artigo original podia ser localizado; e aqueles que, embora apresentando definições explícitas, essas podiam ser localizadas em artigos anteriores de mesmo teor. Essa segunda fase foi iterativamente complementada com buscas no Google Acadêmico que visava coletar artigos que eventualmente não estivessem disponíveis no Portal da CAPES.

Definição foi entendida como "representação de um conceito por meio de um enunciado descritivo, que serve para diferenciá-lo de outros conceitos relacionados", enquanto conceito foi definido como "unidade de conhecimento criada por uma combinação única de características" (International Organization for Standardization, 2000). Finalmente, a descrição de cada termo metacognitivo foi feita por meio de definições operacionais (Cooper, 1982).

Para a produção das definições baseamo-nos no questionamento aristotélico buscando suas quatro causas (material, formal, eficiente e final), perguntando para cada termo em construção: Quê? Quem? Quando? Como? Por quê? Para quê? (Salomom, 1993; Shields, 2016). Assim, por exemplo, para definir o termo "Metacognição" perguntamo-nos e respondemos:

1. O que é? E respondemos: "É um discurso de segundo nível sobre a cognição".

2. Quem? Resposta implícita: O sujeito pensante.

3. Quando? Resposta: "No ato de pensar" (obviamente dispensável na definição)

4. Como? Resposta: "Caracterizada pela: a) Consciência...[...]"

5. Para quê? Resposta: "Intencionalidade e autorregulação [...]"

O estudo procurou espelhar o estado atual da literatura sem pretender avançá-la. Por esse motivo, a forma completa das definições nem sempre esteve presente. Aplicou-se o software Freemind (C para a compilação de todas as definições e termos sob a forma de um mapa mental (Buzan, 2005) visando organizá-las de forma hierárquica.

\section{RESULTADOS: DEFINIÇÕES DE TERMOS METACOGNITIVOS}

METACOGNIÇÃo (MTCG) - Definição: Discurso de segundo nível sobre a cognição (Metcalfe \& Shimamura,
1994; Hacker, 1998). Caracterizada por: a) Consciência sobre os processos cognitivos - Capacidade de explicitar e refletir sobre eventos e processos cognitivos presentes, passados ou futuros; b) Intencionalidade - A ação metacognitiva faz-se com um objetivo; c) Autorregulação - A MTCG é relativamente independente do ambiente externo. São os recursos pessoais que definem o curso de ação do indivíduo, na presença dos fatores ambientais e da tarefa. Esses últimos impõem limites à ação, mas são os fatores pessoais que definem o modo de agir do indivíduo, e d) Multi-nível - É estratificada. No nível inferior, ocorrem os processos cognitivos. Subordinando o nível cognitivo, há o metacognitivo, que o modula segundo suas regras próprias. No seu interior (ou acima), como um meta-nível de terceira ordem, ocorre o processo de auto regulação da MTCG onde, por definição, usa suas próprias regras para manter-se em funcionamento adequado.

A-HABILIDADES METACOGNITIVAS - Definição: Representam as competências necessárias para o controle voluntário sobre seus próprios processos cognitivos por meio do conhecimento processual, expresso pelo uso deliberado das estratégias (Brown, 1978; Efklides, 2008; Zohar \& Ben David, 2009). Objetivo: Participam na resolução de problemas (Zohar \& Ben David, 2009) e na regulação e controle sobre a própria aprendizagem (Veenman \& Elshout, 1999). Contexto: Para serem ativadas, é preciso que haja consciência da fluência do processamento cognitivo e da ocorrência de conflito ou erro (Efklides, Samara, \& Petropoulou, 1999). Como funcionam: São como agentes de ordem superior, que supervisionam e governam o sistema cognitivo, porém, simultaneamente, fazem parte dele, usando ambos os processos de ir e vir constante. Durante a execução de tarefas fazem uso dos conhecimentos específicos a ela, bem como do conhecimento metacognitivo pertinente (Veenman, Hout-Wolters, \& Afflerbach, 2006). Operam na cognição por meio do laço cognitivo de regulação e podem ter ligação com estratégias cognitivas-como ensaio, elaboração e assim por diante - para regular a cognição, bem como estratégias para analisar os requisitos da tarefa e avaliar a resposta (Efklides, 2008). Caracterizam-se como: Estratégias Metacognitivas, definidas como um conjunto de operações mentais cognitivas e interdependentes que tem por função gerenciar as tarefas relativas à cognição e, para tal, podem ser modificadas em resposta a diferentes situações. Essas estratégias e seus desempenhos correspondentes podem ser controlados pelo uso dos conhecimentos específicos a tarefa, bem como do conhecimento metacognitivo pertinente (Efklides, 2008). Incluem estratégias de orientação, planejamento de estratégias, regulação do processamento cognitivo, acompanhamento da execução das ações planejadas, e avaliação do resultado do processamento da tarefa (Veenman \& Elshout, 1999). 
A.1 - Previsão-Definição: Habilidade de permitir o pensar sobre os objetivos de aprendizagem, características adequadas de aprendizagem e tempo disponível. Objetivo: Prever as dificuldades da tarefa e faz com que se trabalhe lentamente em tarefas difíceis e mais rapidamente nas mais fáceis. Estimar ou prever a dificuldade de uma tarefa e usar essa previsão metacognitivamente para regular o compromisso do aprendiz em relação à expectativa de resultado e eficácia. Além disso, a previsão gera relação entre problemas, desenvolve a intuição sobre os pré-requisitos de uma tarefa e distingue entre dificuldades aparentes e reais na resolução de problemas (Desoete, 2008).

A.2 - Planejamento - Objetivo: Pensar, com antecipação, como, quando e o porquê de agir no sentido de obter os seus objetivos, por meio de uma sequência de submetas que conduz à meta principal do problema (Desoete, 2008).

A.3 - Monitoração - Definição: Controle autorregulado de habilidades cognitivas usadas durante $o$ desempenho atual, a fim de identificar problemas e modificar planos (Desoete, 2008).

A.3.1 - Controle-Definição: Sistema de meta-nível que atua sobre os processos cognitivos de nível mais baixo. É, no sentido do fluxo informacional, um processo de baixo para cima (Nelson \& Narens, 1990), como por exemplo, a atribuição de mais tempo de estudo ou o lançamento de algumas estratégias de recuperação (Shimamura, 2000b). Caracteriza-se por: apresentar modelos ideais de funcionamento dos processos cognitivos e ao mesmo tempo conferir a sua adequação aos modelos pré-existentes. Objetivo: Regular por apontar inadequações e os procedimentos corretivos necessários.

A.3.1.1 - Controle executivo das ações - Definição: Conjunto de processos envolvidos na ativação e manipulação da informação na memória de trabalho (Shimamura, 2000a). Incluem tarefas que exigem esforço no processamento cognitivo tais como resolução de conflito, controle inibitório, deteç̧ão de erro e regulação emocional. Conceito: São talvez os blocos fundamentais de que pensadores metacognitivamente sofisticados fazem uso em tarefas como solução de problemas, seleção de estratégias e tomada de decisão. Estudos de neuroimagem mostraram que durante o controle executivo é ativada uma rede de áreas na região frontal do cérebro que inclui os córtices cingulado anterior, o orbitofrontal e o dorsolateral, a área motora suplementar, além de porções dos gânglios basais e do tálamo (Fernandez-Duque, Baird, \& Posner, 2000). Classificação: São quatro os aspectos. Na ativação da informação, há (1) a seleção e a (2) manutenção. Na sua manipulação, há (3) a atualização e (4) o reencaminhamento. São organizados por nível de complexidade, desde o seu aspecto mais rudimentar de controle - a seleção, até o aspecto mais exigente - o reencaminhamento (Shimamura, 2000b).
A.3.1.1.1 - Seleção-Definição: Capacidade de focar a atenção para eventos de estímulo ou ativar as representações de memória. Caracteriza-se por: relacionar-se com o conceito de atenção seletiva. Em situações de conflito, como o Teste de Stroop ${ }^{1}$, o controle deve permitir a seleção de características de determinado estímulo, ao mesmo tempo que a filtragem de outros.

A.3.1.1.2 - Manutenção - Definição: Capacidade de manter a informação ativa na memória de trabalho. Tarefas realizadas pela memória de trabalho tais como as de extensão numérica (Digit Span Task²) fazem uso desse processo (Hitch, Burgess, Towse, \& Culpin, 1996) Conceito relacionado: Memória de curto prazo.

A.3.1.1.3 - Atualização - Modula e reorganiza a atividade na memória de trabalho. Pode ser avaliada pelo $n-$ back $^{3}$ (Kirchner, 1958). Conceito relacionado: Monitoração.

A.3.1.1.4 - Reencaminhamento - Definição: Mudança de um processo cognitivo ou de resposta previstos para o outro. Pode ser avaliado pelo teste de mudança de tarefa ${ }^{4}$. Conceito Relacionado: seleção de deslocamento.

A.3.2 - Regulação - Definição: Processos que coordenam a cognição a partir das informações fornecidas pelo mecanismo de controle. Características: É um sistema de meta-nível que modula os processos cognitivos de nível mais baixo. É, na perspectiva do fluxo informacional, um processo de cima para baixo (Fernandez-Duque et al., 2000).

\footnotetext{
1 "O efeito Stroop (às vezes chamado de teste Stroop) é um resultado da nossa (atenção) vitalidade mental e flexibilidade. O efeito está relacionado com a capacidade de ler na maioria das pessoas a palavras mais rapidamente e automaticamente que eles possam citar cores" (De Young, 2014).

2"As tarefas de extensão de memória (span) diretas referemse à capacidade de um sujeito reproduzir, imediatamente após uma apresentação verbal, uma série de estímulos na sua ordem original. Esses estímulos/itens podem variar entre palavras, números e letras, havendo quem utilize também pseudopalavras. Este tipo de tarefa é considerada uma medida comum de MCP verbal, uma vez que solicita apenas o armazenamento da informação verbal." (Fernandes, 2012)

${ }^{3}$ É um paradigma comumente utilizado para avaliar a memória operacional, indicado para avaliar suas habilidades de manipulação e atualização da informação (Gonçalves-Calado, 2013). Neste teste, os indivíduos devem não só manter a informação, mas também manipular ou atualizar informações. Apresenta-se ao sujeito testado uma sequencia de estímulos, e a tarefa consiste em indicar quando o estímulo atual é igual a um dos $n$ passos anteriores na sequência. $O$ fator de carga $n$ pode ser ajustado para tornar a tarefa mais ou menos difícil (wikipedia, 2014).

${ }^{4}$ Teste que submete o participante a mudanças na execução de múltiplas tarefas individuais. O AST (Attention Switching Task) Testa a habilidade do participante em alternar o foco de sua atenção entre a direção e a localização de uma seta na tela de um computador. É uma medida sensível do estado do lobo frontal e de disfunções executivas (Cognitive Atlas, 2021).
} 
A.3.2.1 - Regulação propriamente dita / A.3.2.1.1 - Atenção Seletiva - Definição: Habilidade em atender a alguma atividade mental em detrimento de outras. Em tarefas com mensagens competitivas, o indivíduo é solicitado a selecionar uma informação e ignorar as demais, ou seja, deverá focar sua atenção no estímulo solicitado e, portanto, deverá recuperar somente uma das informações. Características: Similar à "seleção" (sistema de monitoração), respondendo, no entanto à regulação. Denominado simplesmente como "atenção", é um sistema que sofre um processo longo de desenvolvimento e tem profundas consequências para o desenvolvimento cognitivo e emocional, vontade e consciência, que são temas de grande interesse para os estudiosos da MTCG (Fernandez-Duque et al., 2000). Relacionado ao processo de atenção seletiva, existe a habilidade de figura-fundo, que é a capacidade do indivíduo de identificar a mensagem primária na presença de mensagens competitivas (Garcia, Pereira, \& Fukuda, 2007).

A.3.2.1.2 - Resolução de conflitos - Características: Ocorre na presença de dois ou mais estímulos competitivos e incongruentes. Selecionar uma única dimensão de um estímulo multidimensional é uma tarefa que envolve conflito e, portanto, cria a necessidade de um processo de resolução. Exemplo clássico de conflito é o que ocorre no Teste de Stroop onde cor e palavra competem entre si, sendo necessário haver resolução deste conflito de modo que seja possível identificar a mensagem (Fernandez-Duque et al., 2000).

A.3.2.1.3 - Detecção de erro - Definição: Identificação de discrepâncias entre o objetivo e o produto do processamento, frequentemente considerado um índice de monitoramento da compreensão (Shimamura, 2000b). Características: Fernandez-Duque et al. (2000) adicionam o termo "Correção" afirmando ser comum que os sujeitos cometam erros ao executar uma tarefa, e que indivíduos normais têm a capacidade de avaliar internamente o seu próprio desempenho e detectar os erros, mesmo na ausência de feedback externo. Estudos têm mostrado que, após a deteç̧ão de um erro, sujeitos ajustam a velocidade do seu desempenho para alcançar um nível adequado de precisão.

A.3.2.1.4 - Controle inibitório - Definição: Inibição de respostas competitivas e irrelevantes (Knight, Scabini, \& Woods, 1989; Knight, Staines, Swick, \& Chao 1999). Capacidade de adiar, focar a atenção e reprimir desejos imediatos ou impulsos. Características: Relaciona-se de maneira próxima a traços considerados como prototípicos da capacidade de restrição, a saber: deliberação, controle de impulsos, capacidade de planejar e persistência na consecução de metas distantes (Kochanska, 1997).

A.3.2.1.5 - Planejamento - Definição: Reflexão sobre qual curso de ação é necessário para alcançar uma meta e, como tal, o planejamento é parte da MTCG (Shimamura, 2000b). Características: A ação de planejar requer o estabelecimento tanto de uma meta principal como de uma hierarquia de submetas que devem ser satisfeitas para que o objetivo principal seja obtido. O objetivo principal geralmente orienta as submetas (Fernandez-Duque et al., 2000).

A.3.2.1.6 - Regulação emocional - Definição: Uso das informações de caráter emocional para modificar a resposta. Características: Na maioria das situações, os sinais de feedback têm tanto a informação cognitiva como a emocional. Por exemplo, um sinal de erro pode informar às pessoas que estão indo muito rápido na tarefa, mas também é susceptível que possa desencadear uma emoção negativa. Há evidência de que a valência do feedback leva a uma mudança automática no critério de resposta, mesmo na ausência de qualquer informação cognitiva (Derryberry, 1991). Por exemplo, a apresentação de uma sugestão de valência, por exemplo, uma expressão facial triste, durante uma tarefa contínua de desempenho pode diminuir a resposta e reduzir futuros erros de omissão, mesmo quando sujeitos corretamente acreditam que a sugestão é pouco informativa sobre o seu desempenho (Fernandez-Duque et al., 2000).

A.3.2.1.7 - Memória de trabalho - Definição: Componente cognitivo da memória encarregado dos processos e representações envolvidas na ativação ou armazenamento de informação de maneira temporária. Características: Shimamura (2000a) apresenta o modelo proposto por Baddeley, onde a memória de trabalho é representada por uma central executiva que controla as informações em três "amortecedores" de armazenamento: o laço fonológico, a alça visuo-espacial e o episódico.

A.3.2.2 - Co-regulação e outra regulação da cognição-Definição: Controle da própria cognição seguindo feedback de outra(s) pessoa(s), ou pela orientação direta dada por outra pessoa (outra regulação) (Efklides, 2008).

A.4 - Avaliação (julgamentos) - Definição: Integra o conhecimento e o sentimento metacognitivos para definir o estado atual do resultado e o curso futuro do processamento da tarefa. Características: É uma monitoração da fonte. Nessas tarefas, os indivíduos devem avaliar informações contextuais, tais como se lembrar de quando ou onde tenha ocorrido algum evento ou quem apresentou alguma informação (Johnson, Hashtroudi, \& Lindsay, 1993). Ao avaliar, refletimos sobre os resultados, o entendimento do problema, a adequação do plano, a execução do método de solução, bem como sobre a adequação da resposta dentro do contexto do problema (Vermeer, 1997). Tais reflexões ocorrem depois de um evento ter acontecido (Brown, 1987, citado por Desoete, 2008). Nelas, olhamos para o que fizemos e se isso levou ou não a um resultado 
desejado (Vermeer, 1997). Inclui avaliações de memória que se fazem por meio de duas estratégias metacognitivas (Shimamura, 2000b) apresentadas a seguir em A.4.1 e A.4.2.

A.4.1 - Julgamentos de aprendizagem - Judgment of Learning (JOL) - Definição: Avaliam a aprendizagem presente. Características: Aqui o sujeito se questiona: O quão bem eu aprendi o material? (Nelson \& Narens, 1994).

A.4.2 - Julgamentos de Sentimento de saber - Feeling of knowing (FOK) - Definição: Avaliam o desempenho futuro. Características: Aqui o sujeito se questiona: $\mathrm{O}$ quão bem eu vou me sair em um teste? Ocorrendo durante ou após a fase de aquisição de novos materiais, é um processo analítico exprimindo a capacidade de prever a recuperação de um dado item que no momento não é recuperável (Nelson \& Narens, 1994).

B - EXPERIÊNCIA METACOGNITIVA - Definição: É a interface entre a pessoa e a tarefa. É o que a pessoa tem de consciente e o que ela vai sentir quando se encontrar frente a uma tarefa e quando processar as informações relacionadas a ela (Efklides, 2008). Características: Inclui os sentimentos e julgamentos metacognitivos decorrentes da monitoração de aspectos de processamento ou resultados de tarefas (Efklides, 2006). A experiência tem um caráter totalizante e inclui aspectos racionais, afetivos e sensoriais.

B.1 - Sentimento Metacognitivo - Definição: Segundo Efklides (2008), são informações de caráter afetivo e valor positivo ou negativo percebido pelas pessoas nas situações que vivenciam. Características: São produtos do inconsciente, de processos inferenciais não analíticos. Uma vez que eles aparecem na consciência, fornecem a base de dados para os julgamentos / estimativas analíticas ou controle de decisões.

B.1.1 - Julgamentos/estimativas metacognitivas - Definição: Informam a discrepância entre a resposta e o objetivo. Características: Fazem interface com os sentimentos de dificuldade e de confiança. Incluem a) Julgamento de aprendizagem, b) Estimativa da despesa de esforço para a realização da tarefa, c) Estimativa de tempo necessário ou gasto para a realização da tarefa, d) Estimativa de correção de solução.

B.1.2 - Sentimento de dificuldade - Definição: É a informação sobre a falta de fluência no processamento da tarefa (Efklides, 2006). Características: Surge no contexto da resolução de problemas (Efklides \& Petkaki, 2005). Está associado com o afeto negativo decorrente da falta de fluência devido à interrupção do processo. Indica que a pessoa tem de investir mais esforço para passar mais tempo no processamento de tarefas ou para reorganizar a resposta (Efklides \& Petkaki, 2005). Alerta o indivíduo sobre a necessidade de tomada de decisão porque há conflito de respostas e aumento da probabilidade de erro (Veen \& Carter, 2002). É, portanto, capacidade relevante para a autorregulação do esforço. Monitora o conflito de respostas (Veen \& Carter, 2002) ou a interrupção do tratamento, ou seja, um erro ou falta de resposta disponível (Mandler, 1984, citado por Efklides, 2006).

B.1.3 - Sentimento de confiança - Definição: É informação que aflora ao final do processamento de uma tarefa, resultante do balanceamento entre os sentimentos positivo e negativo percebidos pelo indivíduo sobre essa tarefa (Efklides \& Petkaki, 2005).

B.1.4 - Sentimento de saber - Definição: É o sentimento de caráter positivo que informa a pessoa sobre o conhecimento que ela possui sobre um dado item (Efklides, 2006).

B.1.5 - Sentimento de familiaridade - Definição: É uma informação (afetiva) sobre a ocorrência anterior de um estímulo. Características: Denota a fluência de processamento (Cornoldi, 1998; Efklides, 2008). Está associado a afetos positivos decorrentes da fluência na acessibilidade da respectiva informação (Efklides, 2006).

B.2 - Consciência Metacognitiva - Definição: Autoexplicativo. Características: conhecimento que permite ao aprendiz vivenciar, experimentar ou compreender aspectos ou a totalidade do seu mundo metacognitivo. Inclui a) Características da tarefa, b) Fluência de processamento cognitivo, c) Progresso em direção ao objetivo proposto, d) Esforço despendido no processamento cognitivo, e) Resultado do processamento (Efklides, Kourkoulou, Mitsiou, \& Ziliaskopoulou, 2006; Efklides, 2008).

C-CONHECIMENTO METACOGNITIVO - Definição: É o conhecimento declarativo armazenado na memória, sobre habilidades e estratégias cognitivas, tarefas e, ainda, modelos de processos cognitivos, tais como memória, linguagem e assim por diante (Flavell, 1979; Fabricius \& Schwanenflugel, 1994). Finalidade: O conhecimento metacognitivo faz uso da linguagem como uma ferramenta que permite (King, 1998; Efklides, 2008): a) Comunicar o conteúdo da consciência pessoal para os outros, b) Refletir, tirar conclusões e fazer atribuições sobre as relações entre estados internos e comportamentos observáveis assim como resultados da ação, c) Capacitar as pessoas a analisar e comparar os seus estados mentais subjetivos e conhecimentos com os de outras pessoas. Isto se faz em associação com a reflexão, d) Formular teorias explícitas sobre o conhecimento e a cognição, e) Construir, como resultado dos itens anteriores, um modelo socialmente compartilhado e socialmente negociado da cognição, de si mesmo e dos outros como seres cognitivos. Origem: 0 conhecimento metacognitivo origina-se da integração das informações provenientes do monitoramento da cognição em um nível consciente. Como consequência dá-se o seu enriquecimento, atualização e diferenciação. Resulta da: a) observação do próprio comportamento/ 
ações e o dos outros e seus resultados quando se lida com tarefas específicas em diversos contextos (Fabricius \& Schwanenflugel, 1994), b) conscientização de nossa experiência metacognitiva (Flavell, 1979) e c) comunicação e interação com os outros (Ruffman, Slade, \& Crowe, 2002).

C.1 - Conhecimento Metacognitivo sobre a Tarefa - Definição: É mobilizado durante sua execução e é representado pelas categorias de tarefas e suas características, as relações entre as tarefas, bem como as maneiras com que elas são processadas (Flavell, 1979). Características: Inclui informações sobre: a) A tarefa que estamos executando, b) Ideias ou pensamentos que nós estamos cientes de como lidamos com uma tarefa. Por exemplo, os procedimentos cognitivos que estamos aplicando e c) O conhecimento metacognitivo que recuperamos da memória a fim de processar a tarefa. Por exemplo, conhecimento metacognitivo sobre tarefas e procedimentos que foram utilizados no passado, a comparação da tarefa corrente com outras tarefas no que se refere às suas semelhanças ou diferenças, $e$ assim por diante.

C.2-Conhecimento Metacognitivo sobre as Pessoas - Definição: Como nós ou outros processam as várias tarefas e o quão bons somos nisto e, ainda, o que foi sentido durante o processamento de uma tarefa específica (Flavell, 1979). Isto é, o conhecimento de mim e dos outros enquanto seres cognitivos.

C.3 - Conhecimento Metacognitivo sobre as Estratégias - Definição: Conjunto de informações sobre múltiplas estratégias, bem como as respectivas condições de utilização. Isto é, quando, por que e como a estratégia deve ser usada (Efklides, 2008).

C. 4 - Conhecimento Metacognitivo sobre as Metas - Definição: Objetivos implícitos ou explícitos que impulsionam, mantêm e direcionam o empreendimento cognitivo visando a realização de tarefas ou situações específicas (Ribeiro, 2003; Jou \& Sperb, 2006; Efklides, 2008).

C.5 - Meta-Conhecimento Metacognitivo - Definição: É um conhecimento de segunda mão sobre acontecimentos factuais da cognição e sobre estratégias e/ou procedimentos. É também um conhecimento executivo, representado por regras de produção, ou seja, regras de condição-ação. Isto é, no ato da ação cognitiva, explicita as regras necessárias para a sua execução (Figueira, 2003).

\section{DISCUSSÃO}

O termo metacognição foi proposto para descrever, em crianças, um fenômeno que incluía conhecimento pessoal sobre processos cognitivos, bem como sua monitoração (Flavell, 1979). Desde então, estudos posteriores ampliaram e aprofundaram o conceito; contudo, uma fraca coesão expandiu a incerteza conceitual
(Desoete \& Ozsoy, 2009; Scott \& Levy, 2013b).

Os procedimentos para a construção da taxonomia, dentre eles o de organizar conceitos em um sistema lógico, produziram um constructo que oferece aos pesquisadores a possibilidade de lidar com duas funções da taxonomia. Por um lado, a de alocar e recuperar informação; por outro, ao permitir e facilitar a comunicação. E ainda mais, sua hierarquia inerente torna-a ferramenta de aprendizado para seus usuários (Campos \& Gomes, 2008).

A taxonomia apresentada buscou lidar com os desafios da diversidade de campos de pesquisa e orientações teórico-metodológicas. Os autores não avocam a si a construção de uma nova taxonomia, mas a compilação de rótulos, definições e categorias previamente presentes na literatura, buscando-se manter a neutralidade possível, omitindo-se de criticar o já produzido. Nesse sentido então é que a ênfase deste estudo foi na organização e síntese de material disperso e eventualmente conflitante.

No esforço de harmonização dos conceitos e das definições, os autores proponentes foram obrigados a tomar decisões baseadas no conjunto dos dados obtidos e ainda em suas próprias perspectivas frente aos fenômenos metacognitivos. Sendo assim, há que se entender que se trata de "uma taxonomia" e não de "A taxonomia" metacognitiva. Como qualquer esforço classificatório, não é um ponto final ao conhecimento nele contido.

A taxonomia proposta neste artigo representa um sistema derivado da criação de categorias e rótulos taxonômicos compilados, analisados, interpretados e organizados para o uso em um contexto específico: o do trabalho de identificação de fenômenos metacognitivos obtidos por instrumentos de coleta que permitam a produção de textos tais como entrevistas ou questionários. Assim, há limites para a sua aplicação quando se tratar de outros tipos de produção de dados derivados de procedimentos metodológicos que não produzam discursos verbais ou escritos, como por exemplo, os dados de imagem cerebral nas pesquisas de neurociência.

Dentre os benefícios das definições apresentadas depreende-se que a sua disponibilização organizada permite tanto a presunção de relações entre conceitos e, ao oferecer elementos para uma linguagem comum, facilita a fertilização recíproca de campos de investigação ainda hoje isolados.

O que se alcançou nessa pesquisa foi a apresentação de uma taxonomia seletiva e focada em eventos metacognitivos passíveis de serem identificados em textos escritos com na proposição de definições operacionais que permitam a sua identificação e aplicação em pesquisas que tomem como dado as subjetividades ligadas à linguagem, reiterando que não se pretendeu nem propor novos fenômenos metacognitivos, nem 
reivindicar a originalidade completa de cada definição apresentada.

A reflexão sobre essa taxionomia pode supor certa funcionalidade. As definições foram aglutinadas em três categorias de construtos metacognitivos: Habilidades (A), Experiências (B) e Conhecimento (C). Ao refletir sobre essas categorias e os termos que lhes são subordinados é possível delinear um modelo conceitual em maior nível de abstração.

Na Habilidade Metacognitiva os termos são em muito maior número que nas outras duas categorias. Também verifica-se uma destacada predominância de subtipos ligadas à monitoração (A.3) o que pode indicar um interesse maior dos pesquisadores pela investigação da "metacognição em ação" e em particular pelas habilidades de monitoramento de processos cognitivos, o que converge para uma das mais reconhecidas finalidades da metacognição.

Porém, diferentes desta, as outras duas, Experiência e Conhecimento reduzem-se a poucos termos. E ainda mais, também diversamente, os termos são mais estáticos e quase que diretamente descritivos de eventos instantâneos. Por exemplo, um "Conhecimento Metacognitivo sobre a Tarefa (C1)" apenas explicita um conjunto de informações sobre um quefazer específico. Já a "Monitoração (A3)" refere-se a um processo dinâmico de controle da cognição regulado pelo próprio indivíduo.

Por isto e em uma interpretação teleológica, é possível pensar em um modelo em que a Experiência e o Conhecimento Metacognitivos constituam-se em fornecedores de matéria prima informacional para que a Habilidade Metacognitiva possa cumprir sua função de gerenciamento cognitivo. Não se trata de uma hierarquia, enfatize-se, mas de um sistema interdependente, mesmo que finalista.

\section{REFERÊNCIAS}

Brown, A. L. (1978). Knowing when, where, and how to remember: A problem of metacognition. Advances in instructional psychology, 1(1), 77-165.

Buzan, T. Mapas Mentais e Sua elaboração: Um sistema definitivo de pensamento que transformará sua vida. Trad. Euclides Luiz Calloni; Cleusa Margô Wosgrau. 1. ed. São Paulo: Cultrix, 2005.

Campos, M. L. de A.; Gomes, H. E. (2008). Taxonomia e Classificação - o princípio de categorização. DataGramaZero - Revista de Ciência da Informação, 9(4).

INTERNATIONAL ORGANIZATION FOR STANDARDIZATION. ISO 1087 - Terminology work - Vocabulary - Part 1 - Theory and application. Geneva, Switzerland: ISO-International Organization for Standardization, 2000. Disponível em: $<$ https://edisciplinas.usp.br/pluginfile.php/312608/mod_ resource/content/1/ISO_1087-1_2000_PDF_version_(en_ fr)_CPDF.pdf>.

Cooper, H. M. (1982). Scientific Guidelines for
Conducting Integrative Research Reviews. Review of Educational Research, 52(2), 291-302. https://doi. org/10.3102/00346543052002291

Cooper, Harris M. (1988). Organizing knowledge syntheses: A taxonomy of literature reviews. Knowledge in Society, 1(1), 104-126. https://doi.org/10.1007/BF03177550

Cordeiro, A. M.; Oliveira, G. M. de; Rentería, J. M.; Guimarães, C. A. (2007). Revisão sistemática: Uma revisão narrativa. Revista do Colégio Brasileiro de Cirurgiões, 34(6), 428-431. https://doi.org/10.1590/S0100-69912007000600012

Cornoldi, C. (1998). The impact of Metacognitive Reflection on Cognitive Control. In: G. Mazzoni; T. O. Nelson (Eds.), Metacognition and Cognitive Neuropsychology (p. 144). Mahwah, New Jersey: Lawrence Erlbaum Associates.

De Young, R. (2014). Using the Stroop effect to test our capacity to direct attention: A tool for navigating urgent transitions. Recuperado em 06 jul. 2014, de http://www.snre.umich. edu/eplab/demos/st0/stroopdesc.html

Derryberry, D. (1991). The immediate effects of positive and negative feedback signals. Journal of Personality and Social Psychology, 61(2), 267-278.

Desoete, A. (2008). Multi-method assessment of metacognitive skills in elementary school children: How you test is what you get. Metacognition and Learning, 3(3), 189-206. https://doi.org/10.1007/s11409-008-9026-0

Desoete, A.; Ozsoy, G. (2009). Introduction: Metacognition, More than the Lognes Monster?. International Electronic Journal of Elementary Education, 2(1), 1-6.

Efklides, A.; Samara, A.; Petropoulou, M. (1999). Feeling of difficulty: An aspect of monitoring that influences control. European journal of psychology of education, 14(4), 461-476.

Efklides, A. (2006). Metacognition and affect: What can metacognitive experiences tell us about the learning process? Educational Research Review, 1(1), 3-14. https:// doi.org/10.1016/j.edurev.2005.11.001

Efklides, A. (2008). Metacognition-Defining Its Facets and Levels of Functioning in Relation to Self-Regulation and Co-regulation. European Psychologist, 13(4), 277-287. https://doi.org/10.1027/1016-9040.13.4.277

Efklides, A; Kourkoulou, A.; Mitsiou, F.; Ziliaskopoulou, D. (2006). Metacognitive knowledge of effort, personality factors, and mood state: Their relationships with effortrelated metacognitive experiences. Metacognition and Learning, 1(1), 33-49. https://doi.org/10.1007/s11409006-6581-0

Efklides, A; Petkaki, C. (2005). Effects of mood on students' metacognitive experiences. Learning and Instruction, 15, 415-431.

Fabricius, W. V.; Schwanenflugel, P. J. (1994). The older child's theory of mind. In: A. Demetriou; A. Efklides (Eds.), Advances in psychology (Vol. 106, p. 111-132). ScienceDirect. Recuperado de https://www.sciencedirect. com/bookseries/advances-in-psychology/vol/106/suppl/C

Fernandes, P. A. G. (2012). Memória e envelhecimento: A 
influência da idade no declínio da memória de trabalho (Dissertação de Mestrado). Universidade Católica Portuguesa, Lisboa. Recuperado de http://repositorio.ucp. pt/handle/10400.14/8846

Fernandez-Duque, D.; Baird, J. A.; Posner, M. I. (2000). Executive Attention and Metacognitive Regulation. Consciousness and Cognition, 9(2), 288-307. https://doi. org/10.1006/ccog.2000.0447

Figueira, A. P. C. (2003). Metacognição e seus contornos. Revista Iberoamericana de Educación, $\mathrm{s} / \mathrm{n}$. Recuperado de https://rieoei.org/historico/deloslectores/446Couceiro.pdf

Flavell, J. H. (1976). Metacognitive Aspects of Problem Solving. In: The Nature of Inteligence. (pp. 231-235). 1. ed. Hillsdale N.J.: Lawrence Erlbaum Associates.

Gagnière, L.; Betrancourt, M.; Détienne, F. (2012). When metacognitive prompts help information search in collaborative setting. Revue Européenne de Psychologie Appliquée/European Review of Applied Psychology, 62(2), 73-81. https://doi.org/10.1016/j.erap.2011.12.005

Garcia, V. L.; Pereira, L. D.; Fukuda, Y. (2007). Atenção seletiva: PSI em crianças com distúrbio de aprendizagem. Revista Brasileira de Otorrinolaringologia, 73(3), 404-411. https:// doi.org/10.1590/S0034-72992007000300017

Gonçalves-Calado, V. T. (2013). Desempenho de indivíduos acometidos por traumatismo cranioencefálico no teste $n$-back auditivo (Dissertação de Mestrado). Faculdade de Medicina da Universidade de São Paulo, São Paulo. Recuperado de https://teses.usp.br/teses/ disponiveis/5/5162/tde-03012014-150743/publico/ VanessaTomeGoncalvesCalado.pdf

Hacker, D. J. (1998). Definitions and empirical foundations. In: Hacker, D. J.; Dunlosky, J.; Graesser, A. C. (Eds.), Metacognition in educational theory and practice (p. 8-20). Mahwah, New Jersey: Lawrence Erlbaum Associates.

Hitch, G. J.; Burgess, N.; Towse, J. N.; Culpin, V. (1996). Temporal Grouping Effects in Immediate Recall: A Working Memory Analysis. Quarterly Journal of Experimental Psychology: Section A, 49(1), 116-139. https://doi. org/10.1080/027249896392829

Johnson, M. K.; Hashtroudi, S.; Lindsay, S. (1993). Source Monitoring. Psychological Bulletin, 114(1), 3-28, 1993.

Jou, G. I. de.; Sperb, T. M. (2006). A metacognição como estratégia reguladora da aprendizagem. Psicologia: reflexão e crítica., 19(2), 177-185.

King, A. (1998). Transactive peer tutoring: Distributing cognition and metacognition. Educational Psychology Review, 10(1), 57-74. Recuperado de http://link.springer. com/article/10.1023/A:1022858115001

Kirchner, W. K. (1958). Age differences in short-term retention of rapidly changing information. Journal of Experimental Psychology, 55(4), 352-358. https://doi.org/10.1037/ h0043688

Knight, R. T.; Staines, W. R.; Swick, D.; Chao, L. L. (1999). Prefrontal cortex regulates inhibition and excitation in distributed neural networks. Acta psychologica, 101(2),
159-178. https://doi.org/10.1016/S0001-6918(99)00004-9

Knight, R. T.; Scabini, D.; Woods, D. L. (1989). Prefrontal cortex gating of auditory transmission in humans. Brain Research, 54(2), 338-342. https://doi.org/10.1016/00068993(89)91381-4

Kochanska, G. (1997). Multiple pathways to conscience for children with different temperaments: From toddlerhood to age 5. Developmental psychology, 33(2), 228. https:// doi.org/10.1037/0012-1649.33.2.228

Metcalfe, J.; Shimamura, A. P. (1994). Preface. In: J. Metcalfe; A. P. Shimamura (Eds.), Metacognition: Knowing about knowing. Cambridge, MA: The MIT Press.

Moritz, S.; Lysaker, P. H. (2018). Metacognition - What did James $\mathrm{H}$. Flavell really say and the implications for the conceptualization and design of metacognitive interventions. Schizophrenia Research, 201, 20-26. https:// doi.org/10.1016/j.schres.2018.06.001

Wikipedia (2014). N-back. Recuperado em 06/12/2014, de http://en.wikipedia.org/w/index.php?title=Nback\&oldid $=635820870$

Nelson, T. O.; Narens, L. (1990). Metamemory: A Theoretical Framework and new findings. In: G. H. Bower (Ed.), The Psychology of Learning and Motivation: Advances in Research and Theory (Vol. 26, p. 125-174). San Diego: Elsevier.

Nelson, T. O.; Narens, L. (1994). Why Investigate Metacognition. In J. Metcalfe; A. P. Shimamura (Eds.), Metacognition knowing about knowing. (pp. 1-25). Cambridge, MA: MIT Press.

Pereira, M. G. (2012). Artigos científicos: Como redigir, publicar e avaliar. Rio de Janeiro: Editora Guanabara Koogan.

Ribeiro, C. (2003). Metacognição: Um Apoio ao Processo de Aprendizagem. Psicologia: Reflexão e Crítica, 16(1), 109116. https://doi.org/10.1590/S0102-79722003000100011

Rother, E. T. (2007). Revisão sistemática X revisão narrativa. Acta Paulista de Enfermagem, 20(2), v-vi. https://doi. org/10.1590/S0103-21002007000200001

Ruffman, T.; Slade, L.; Crowe, E. (2002). The relation between children's and mothers' mental state language and theoryof-mind understanding. Child development, 73(3), 734-751. https://doi.org/10.1111/1467-8624.00435

Salomom, D. V. (1993). Como fazer uma monografia (2ª ed). São Paulo: Martins Fontes.

Scott, B. M.; Levy, M. (2013a). Metacognition: Examining the Components of a Fuzzy Concept. Educational Research EJournal, 2(2), 120-131. https://doi.org/10.5838/ erej.2013.22.04

Scott, B. M.; Levy, M. G. (2013b). Metacognition: Examining the components of a fuzzy concept. Educational Research eJournal, 2(2), 120-131. DOI: 10.5838/erej.2013.22.04

Shields, C. (2016). Aristotle. In E. N. Zalta (Ed.), The Stanford Encyclopedia of Philosophy (Winter 2016 edition). Metaphysics Research Lab, Stanford University. Recuperado de https://plato.stanford.edu/archives/win2016/entries/ 
aristotle/

Shimamura, A. P. (2000a). The role of the prefrontal cortex in dynamic filtering. Psychobiology, 28(2), 207-218.

Shimamura, A. P. (2000b). Toward a Cognitive Neuroscience of Metacognition. Consciousness and Cognition, 9(2), 313-323. https://doi.org/10.1006/ccog.2000.0450

Tarricone, P. (2011). The Taxonomy of Metacognition. Psychology Press Kindle Edition: Amazon.com.

Attention Switching Task. In: Cognitive Atlas. [s.l.: s.n., s.d.]. Disponível em: <https://www.cognitiveatlas.org/task/id/ trm_4f241614d4a25/>. Acesso em: 27 ago. 2021.

Veen, V.; Carter, C. S. (2002). The Timing of Action-Monitoring Processes in the in the Anterior Cingulate Cortex. Journal of Cognitive Neuroscience, 14(4), 593-602.

Veenman, M.; Elshout, J. J. (1999). Changes in the relation between cognitive and metacognitive skills during the acquisition of expertise. European Journal of Psychology of Education, 14(4), 509-523.

Veenman, M. V. J.; Hout-Wolters, B. H. A. M.; Afflerbach, P. (2006). Metacognition and learning: Conceptual and methodological considerations. Metacognition and Learning, 1(1), 3-14. https://doi.org/10.1007/s11409006-6893-0

Vermeer, H. J. (1997). Sixth-grade students' mathematical problem-solving behavior: Motivational variables and gender differences (Tese de doutorado). Leiden University. Recuperado de https://scholarlypublications. universiteitleiden. $\mathrm{nl} /$ handle/1887/10157

Waltz, C. F.; Strickland, O. L.; Lenz, E. R. (2016). Measurement in Nursing and Health Research (5a ed). Springer Publishing Company. https://doi.org/10.1891/9780826170620

Zohar, A.; Ben David, A. (2009). Paving a clear path in a thick forest: A conceptual analysis of a metacognitive component. Metacognition and Learning, 4(3), 177-195. https://doi.org/10.1007/s11409-009-9044-6

Recebido: 05 de junho de 2019

Aprovado: 11 de março de 2020 